\title{
Enduring Effects of Chronic Corticosterone Treatment on Spatial Learning, Synaptic Plasticity, and Hippocampal Neuropathology in Young and Mid-Aged Rats
}

\author{
Shari R. Bodnoff, ${ }^{1}$ Aaron G. Humphreys, ${ }^{2}$ Jennifer C. Lehman, ${ }^{2}$ David M. Diamond, ${ }^{2,4}$ Gregory M. Rose, ${ }^{2,3,4}$ and \\ Michael J. Meaney' \\ ${ }^{1}$ Developmental Neuroendocrinology Laboratory, Douglas Hospital Research Center, Department of Psychiatry, McGill \\ University, Montreal, Canada H4H 1R3; ${ }^{2}$ Department of Pharmacology and ${ }^{3}$ Neuroscience Training Program, University of \\ Colorado Health Sciences Center, Denver, Colorado 80262; and ${ }^{4}$ Medical Research Service, Veterans Affairs Medical \\ Center, Denver, Colorado 80220
}

\begin{abstract}
Prolonged treatment with stress levels of corticosterone has been reported to produce changes in the hippocampus. In the experiments reported here, we examined for functional and morphological consequences of this treatment. First, young adult or mid-aged male Long-Evans rats were treated for either 1 or 3 months with corticosterone, at a dose sufficient to mimic the elevated hormone levels observed following exposure to mild stress. Two weeks following the termination of treatment, the animals were tested in the Morris water maze to assess spatial learning. No behavioral deficits were observed after 1 month of treatment. A 3 month treatment period also had no effect in young rats, but produced a learning impairment in the mid-aged rats. We then examined whether the effect of elevated corticosterone in mid-aged animals could be produced by a physiological stressor. Mid-aged rats were maintained for 6 months under conditions of low or high social stress. Six months of exposure to high social stress produced significant spatial learning impairments in the Morris water maze. These effects were absent in high social stress animals that had been previously adrenalectomized (with low-level corticosterone replacement), suggesting that elevated glucocorticoid levels mediate the effects of stress on spatial memory in older animals. In a final experiment, mid-aged rats were treated with corticosterone at levels that mimicked those naturally occurring at the diurnal peak (medium-B: 12-17 $\mu \mathrm{g} / \mathrm{dl}$ ) or in response to stress (high-B: $25-32 \mu \mathrm{g} / \mathrm{dl}$ ). Only rats exposed to high levels of corticosterone demonstrated impaired performance in the Morris water maze. Subsequent electrophysiological studies revealed significantly reduced hippocampal synaptic plasticity in both medium-B and high-B
\end{abstract}

\footnotetext{
Received Dec. 10, 1993; revised May 17, 1994; accepted May 26, 1994.

This research was supported by Grants AG09488 to M.J.M. and AG10755 to G.M.R. from the National Institute on Aging, Grant ONR N00014-91-J-1753 to D.M.D. and by funding from the Veterans Affairs Medical Research Service to G.M.R M.J.M. holds an "MRC. Scientist" career award from the Medical Research Council of Canada (MRCC); S.R.B. was supported by a graduate fellowship from the MRCC. The Aging Research Program of the Douglas Hospital Research Center is generously supported by a grant from ALCAN Ltd. Thanks are due to Ms. Carla Frenchko for tireless assistance with typing and editing of the manuscript.

Correspondence should be addressed to Dr. G. M. Rose, Medical Research (151), VAMC, 1055 Clermont Street, Denver, CO 80220

Copyright (C) 1995 Society for Neuroscience $0270-6474 / 95 / 150061-09 \$ 05.00 / 0$
}

animals. Cell counts indicated no significant changes in neuron density in the CA1 and CA3 pyramidal cell layer in either group of the corticosterone-treated rats. Taken together, the data demonstrate that long-term exposure to elevated corticosterone levels resulted in spatial learning deficits in midaged, but not young, rats. Further, these impairments do not appear to have been the consequence of hippocampal neuron loss.

[Key words: glucocorticoids, place learning, memory, hippocampal plasticity, long-term potentiation, aging]

Activation of the hypothalamic-pituitary-adrenal (HPA) axis represents a major component of an organism's defensive response to stimuli that threaten homeostasis. Elevated plasma levels of glucocorticoids (principally corticosterone in the rat and cortisol in primates), along with adrenomedullary catecholamines, are critical for initiating the peripheral catabolic processes required for mobilizing energy reserves to meet the metabolic demands imposed by the stressor (Baxter and Tyrell, 1987). Although short-term glucocorticoid responses to stress are adaptive, chronic exposure to elevated glucocorticoid concentrations contributes to the onset of pathology, including an increased resistance to insulin (and the risk of developing type II diabetes), hypertension, hypercholesterolemia, arteriosclerosis, and immunosuppression (see Munck et al., 1984; Baxter and Tyrell, 1987; Brindley and Rolland, 1989). In addition, prolonged exposure to elevated glucocorticoid levels has been shown to promote neuron loss under a number of conditions, including aging (see Sapolsky, 1990).

Changes in HPA function often occur with aging in the rat. Commonly, these changes include increases in plasma ACTH (Tang and Phillips, 1978; Meaney et al., 1992) and corticosterone concentrations (Hess and Riegle, 1970; Sencar-Cupovic and Milkovic, 1976; Landfield et al., 1978b; Tang and Phillips, 1978; Brett et al., 1983; Sapolsky et al., 1983; DeKosky et al., 1984; Meaney et al., 1992; but see Sontag et al., 1987). In addition to the age-related changes in basal HPA function, senescent animals also exhibit an exaggerated HPA response to stress. While peak HPA responses remain largely unaffected, corticosterone levels remain elevated for significantly longer periods of time compared with young adult animals (see Sapolsky et al., 1986, for a review). Together, these findings suggest that aged 
animals exhibiting HPA dysfunction are exposed to higher cumulative doses of glucocorticoids in the later phases of life.

HPA dysfunction and glucocorticoid hypersecretion have been implicated in the loss of neurons that is generally observed during aging (see Coleman and Flood, 1987). Landfield et al. (1978b) reported that the degree of pathology in the hippocampus was positively correlated with adrenocortical activity, and specifically suggested that elevated glucocorticoids may play a role in the hippocampal pathology associated with aging. Moreover, adrenalectomy at mid-age (with basal level corticosterone replacement) was found to attenuate both the hippocampal neuron loss and cognitive impairments that were observed in the intact aged rat (Landfield et al., 1981). Issa et al. (1990) examined HPA function in aged rats that were tested for spatial learning impairments. They found increased basal and stressinduced HPA activity in cognitively impaired aged rats compared to either cognitively unimpaired aged animals or young adult controls. In these studies, the presence of the cognitive impairments (and increased HPA activity) was associated with hippocampal neuron loss. Sapolsky et al. (1985) exposed young Fischer 344 rats to stress-like levels of corticosterone for three months and comparcd these animals to old ( 28 months) untreated controls. The two groups exhibited similar anatomical sequelae, including a significant loss of large neurons in CA3 and increases in the density of microglia. Thus, exposure to high, but still physiological, concentrations of corticosterone appeared to accelerate the brain aging process in young rats. These findings support the possibility that glucocorticoid-induced hippocampal neuron loss could provide the basis for cognitive impairments in later life (see Landfield et al., 1978b; Issa et al., 1990).

However, exposure to elevated concentrations of glucocorticoids can also alter cognitive function in a manner that appears to be unrelated to neuron loss. In these cases, the impairments occur during elevated steroid exposure and are reversible. There are several reports (e.g., Martignini et al., 1992) of impaired cognitive ability associated with elevated glucocorticoid levels of either endogenous (e.g., Cushing's syndrome, depression; Whelan et al., 1980; Starkman et al., 1981, 1992; Rubinow et al., 1984) or exogenous (glucocorticoid therapy; Hall et al., 1979; Ling et al., 1981; Varney et al., 1984; Martignini et al., 1992) origin. These conditions are also associated with evidence of cerebral atrophy (Momose et al., 1971; Bentson et al., 1978; Okuno et al., 1980), and the severity of damage is related to the daily dosage of steroid. Both the steroid-induced atrophy (Bentson et al., 1978; Okuno et al., 1980) and cognitive disturbances (Ling et al., 1981; Martignini et al., 1992) are reversed following a reduction in glucocorticoid levels. Due to their reversibility, it is unlikely that these effects could involve neuron loss.

Mechanistic evidence for the importance of glucocorticoids in hippocampal function has emerged from studies using in vivo and in vitro models of hippocampal long-term potentiation (LTP), an electrophysiological model of learning and memory (see Madison et al., 1991, for a review). Elevated corticosterone levels are associated with impaired LTP (Foy et al., 1987; Pavlides et al., 1993) and primed burst (PB) potentiation (Bennett et al., 1991; Diamond et al., 1992), the latter a threshold form of LTP in which physiologically patterned electrical stimuli mimics the normal cellular firing patterns within the hippocampus (Rose and Dunwiddie, 1986). These findings suggest that elevated glucocorticoids can decrease hippocampal synaptic plasticity, perhaps thereby interfering with cognitive processes.
To date, the residual effects of long-term exposure to elevated glucocorticoid concentrations have not been extensively examined. In this series of studies, we have examined the consequences of a prolonged period of elevated corticosterone levels upon spatial learning, hippocampal plasticity and neuropathology in adult and mid-aged Long-Evans rats. Effects on behavior and synaptic plasticity were assessed several weeks following the termination of the treatment. The results of these studies indicate that an extended period of elevated glucocorticoid levels causes behavioral and electrophysiological impairments that are similar to those seen in aged rats, and that such sequelae can occur in the apparent absence of hippocampal neuron loss.

\section{Materials and Methods}

Animals. Male Long-Evans rats, at 2 or 9 months of age, were obtained from Charles River (Raleigh, NC) and were housed locally for 3 months. Thus, at the beginning of the experiments, the young adult animals werc 5 months, and the mid-aged rats were 12 months, of age. The animals were maintained on a 12:12 light/dark schedule (lights on at 0800 ), with free access to food (Purina Rat Chow) and water. The health of the animals was monitored regularly, and any animals with overt signs of chronic respiratory distress or tumors were removed from the study. All experimental procedures were conducted in accordance with the guidelines set forth by the Canadian Council on Animal Care and the U.S. National Institutes of Health. Separate groups of rats were used for each of the experiments described.

Steroid pellets and implantation. For experiment 1, corticosterone (4pregnene, $11 \beta,-21$-diol-3,20-dione; Sigma, St. I ouis, MO) or cholesterol ( 5 -cholestene- $3 \beta$-ol; Sigma, St. Louis, MO) pellets were formed by slowly heating the powders in a shallow beaker over a low gas flame to the point when the liquefied steroids could be poured into embedding molds (catalog \#106, Ted Pella Inc., Redding, CA) to solidify. The pellets weighed approximately $100 \mathrm{mg}$ each. Young adult and mid-aged rats were randomly assigned to receive either the corticusterone or cholesterol treatment for either 1 or 3 months. The animals were anesthetized with Metofane (methoxyflurane; Pitman-Moore, Washington Crossing, $\mathrm{NJ}$ ) and the pellets were implanted subcutaneously around the area of the nape of the neck. Preliminary measures of plasma titers, as determined by corticosterone radioimmunoassay (see below) indicated that cholesterol pellets did not alter basal corticosterone values, but two 100 $\mathrm{mg}$ corticosterone pellets were sufficient to produce elevated levels for approximately $10 \mathrm{~d}$ (declining from $41 \pm 2 \mu \mathrm{g} / \mathrm{dl}$ on day 1 to $18 \pm 3$ $\mu \mathrm{g} / \mathrm{dl}$ on day 10 , with an a verage of $30 \pm 3 \mu \mathrm{g} / \mathrm{dl}$ per day). The formation of scar tissue around the pellets was believed to be the major contributing factor to the decline in plasma corticosterone levels over time. The pellets were replaced twice for the 1 month and eight times for the 3 month treatment groups. A different implantation site was used for each replacement pellet.

For experiment 3, corticosterone pellets were purchased from Innovative Research of America (Toledo, OH; $100 \mathrm{mg}$; catalog \#NG-111); these pellets deliver a constant level of steroid for 3 months (veritied in pilot studies in our lab). Cholesterol pellets (\#NC-111) were used in control animals. Weights of the animals were monitored regularly, and additional $10 \mathrm{mg}, 4$ week release pellets (\#G-111) were implanted to compensate for every $10 \%$ increase in body weight. The mid-aged rats in experiment 3 were randomly assigned to receive either medium- or high-corticosterone treatment, or cholesterol, for 3 months. Corticosterone radioimmunoassays on blood samples taken approximately 2.5 months following implantation indicated that two pellets produced a range of plasma corticosterone between 12 and $17 \mu \mathrm{g} / \mathrm{dl}$ (medium-B), while four pellets produced levels in the range of 23-32 $\mu \mathrm{g} / \mathrm{dl}$ (high-B). We observed no mortality in the animals as a result of the corticosterone treatment. After 3 months all pellets were removed and the rats were given a 2 week recovery period prior to behavioral testing to allow for clearance of the steroid and healing of the incision wounds. No scarring was observed with the use of the slow release pellets.

Chronic stress. The chronic social stress paradigm used in these studies was developed by Mormede et al. (1990), who showed that males rats maintained in an unstable social group and cohabitating with females show elevated basal corticosterone levels, with little evidence for adaptation over an extended testing period. In this study 10-month-old male rats were randomly assigned to one of three conditions. (1) Control 
condition: animals were housed four per cage for the duration of the experiment. (2) Stress condition: animals were housed in groups containing two females and two males, and three times weekly the males were rotated according to a quasi-random schedule, such that the same two males were never paired for two consecutive rotations. (3) Stress $+A D X$ condition: animals were adrenalectomized prior to the social stress treatment and implanted with a single $100 \mathrm{mg}$ corticosterone pellet (Innovative Research Advances, Toledo, $\mathrm{OH}$ ) designed to provide basal $(5-6 \mu \mathrm{g} / \mathrm{dl})$ corticosterone levels over a three-month period. The rats were maintained under these conditions for 6 months.

Approximately 5 months into the stress treatment, plasma corticosterone levels were determined in tail blood samples taken at various times over the diurnal cycle (see Meaney et al., 1989, 1992) to verify the effects of the social stress manipulation on HPA activity. At the end of the stress treatment all animals were housed in stable groups of four animals per cage for a 3 week period prior to behavioral testing in the Morris water maze.

Corticosterone radioimmunoassay. Plasma corticosterone was measured using the radioimmunoassay of Krey et al. (1975), using $1 \mu \mathrm{l}$ of plasma and a highly specific corticosterone antiserum (B3-163, Endocrine Sciences, Tarzana, CA), with ${ }^{3} \mathrm{H}$-corticosterone $(88.0 \mathrm{Ci} / \mathrm{mmol}$; New England Nuclear) as tracer. The minimum level of detection of the assay was $10 \mathrm{pg} / \mathrm{ml}$. The antiserum cross-reacts slightly with deoxycorticosterone $(\sim 4 \%)$, but not with cortisol, aldosterone, and progesterone $(<1 \%)$. The intra- and interassay coefficients of variation were $8.9 \%$ and $11.2 \%$, respectively.

Morris water maze. A description of the behavioral apparatus has been described previously (Issa et al., 1990). Briefly, the animals were required to find a submerged platform in a $1.6-\mathrm{m}$-diameter pool of opaque water using only distal spatial cues available within the testing room. The animals were given 20 trials over 10 consecutive days $(2$ trials per day, $10 \mathrm{sec}$ intertrial interval) with the platform submerged. At the end of the testing period rats were given a $30 \mathrm{sec}$ probe trial, in which the platform was removed, to determine spatial bias for the platform location. Finally, all animals werc given five trials in which the platform was raised $2 \mathrm{~cm}$ above the water level (visually cued condition). Visible platform training was used to ensure that impairments were not related to sensorimotor impairments. For all tests, the swim paths were recorded with a digitized television system mounted over the center of the pool and connected to a Videomex-V Image Motion Computer (Columbus Instruments, Columbus, $\mathrm{OH}$ ). Behavioral testing was performed between 1000 and $1600 \mathrm{hr}$.

Electrophysiology. Following the completion of behavioral testing, all rats from experiment 3 were transported in insulated packing crates to the VA Medical Center in Denver, Colorado, for the electrophysiological procedures. The animals were given at least 3 weeks to acclimate to altitude and the new colony room. The day before recording, animals were taken from the vivarium and brought to the testing room. Food and water were removed overnight to prevent fluid accumulation in the lungs while under anesthesia. On the recording day, atropine methyl nitrate was administered $(0.2 \mathrm{mg} / \mathrm{kg}$, i.p.) and rats were anesthetized with an intraperitoneal injection of sodium pentobarbital $(50 \mathrm{mg} / \mathrm{kg}$ in $0.9 \% \mathrm{NaCl}$ ). Supplemental injections of anesthetic were given as necessary throughout the experiment to maintain a stable surgical plane. The rats were placed in a stereotaxic apparatus with the skull oriented in the horizontal plane. The skin overlying the skull was retracted, and the bone and dura above the right dorsal hippocampus and the left side of the ventral hippocampal commissure were removed.

Extracellular recordings of stimulus evoked field potentials in the right dorsal hippocampus were made using capillary glass micropipettes filled with $2 \mathrm{M}$ saline $(0.75-1.25 \mathrm{M} \Omega)$, which were lowered into the $\mathrm{CA} 1$ pyramidal cell layer (coordinates from bregma: AP $-4.0 \mathrm{~mm}$, ML 2.5 $\mathrm{mm}$, DV $1.8-2.3 \mathrm{~mm}$ ). A stimulating clectrode, consisting of a $125-$ $\mu \mathrm{m}$-diameter stainless steel wire insulated with Teflon except at the tip, was lowered into the ventral hippocampal commissure (AP $-2.0 \mathrm{~mm}$, $\mathrm{ML}-1.0 \mathrm{~mm}, \mathrm{DV} 2.8-3.2 \mathrm{~mm}$ ) to activate commissural afferents to the hippocampus. Stimulus pulses were $150-\mu \mathrm{sec}$-duration, cathodal constant current pulses delivered with respect to an indifferent electrode wire located over the posterior cortex (AP $-8.0 \mathrm{~mm}, \mathrm{ML}-4.0 \mathrm{~mm}$ ). A separate recording reference wire, connected to ground, was placed over the frontal cortex (AP $4.0 \mathrm{~mm}$, ML $3.0 \mathrm{~mm}$ ). Electrode positions were optimized electrophysiologically to record the maximal positive field excitatory postsynaptic potential (EPSP) with superimposed population spike (PS). PS amplitude, measured at the midpoint of a tangent connecting the preceding and following positivity of the field EPSP, was automatically recorded by a microcomputer.
The stability of baseline recording was monitored for a least $10 \mathrm{~min}$ before initiating data acquisition procedures. Following this period, population spike measurements were taken from responses evoked by single pulse stimuli given at $30 \mathrm{sec}$ intervals for $5 \mathrm{~min}$. After this, a patterned stimulus train was delivered. This treatment, termed primed burst (PB) stimulation, consisted of a single "priming" stimulus followed $170 \mathrm{msec}$ later by four pulses at $200 \mathrm{~Hz}$ (five pulses total). PB simulation was designed to mimic the complex spike and theta rhythm activity that occurs in the hippocampus (Rose and Dunwiddie, 1986). PB stimulation was applied using the same pulse duration and stimulation intensity as was used to evoke baseline responses. Population spike amplitude was monitored for 20 min following the PB stimulation.

Hippocampal cell counting. Following behavioral and electrophysiological evaluation, the rats were given a lethal injection of sodium pentobarbital and were perfused intracardially with $0.9 \% \mathrm{NaCl}$, followed by $10 \%$ formalin. The brains were removed and stored in $10 \%$ formalin for 1 week, and then placed into a $20 \%$ sucrose/formalin solution for cryoprotection. Cell counting was performed on 30- $\mu \mathrm{m}$-thick, cresyl violet-stained sections of dorsal hippocampus as previously described (Issa et al., 1990). Briefly, raw cell counts were obtained using a $250 \times$ $250 \mu \mathrm{m}$ grid over a $400 \times$ magnified section with a light microscope. Three to five sections of dorsal hippocampus (corresponding to Plates 21 or 22 of the rat brain atlas of Paxinos and Watson, 1982) were analyzed for each animal. Raw cell counts of the pyramidal cell fields were transformed into measures of neuron density per $0.1 \mathrm{~mm}^{2}$ using the method of Abercrombie (1946) to correct for split cell artifacts. Cell size was estimated by measuring the diameter of randomly selecting neurons from both areas CAl and CA3. The length of the cell fields was determined using camera lucida tracings, and the rostrocaudal extent of the dorsal hippocampus was estimated by counting the number of sections necessary to span between Plates 19 and 23 of the Paxinos and Watson atlas. These measurements were made to provide information as to whether potential differences in neuronal density were due to changes in the dimensions of the cell fields.

Statistical analysis. Unlcss othcrwise statcd, the behavioral data were analyzed using a repeated-measures analysis of variance with Tukey post hoc tests performed when appropriate. Statistical analyses of the electrophysiological data were performed using a multivariate analysis of variance (MANOVA) with repeated measures with Tukey post hoc tests.

\section{Results}

\section{Experiment 1: chronic corticosterone in young and mid-aged} rats

Morris water maze. In these experiments, the data were analyzed over days by collapsing across blocks of two trials within days. For the 1 month treatment groups, analysis of the latency (Fig. $1 a$ ) and distance (Fig. $1 b$ ) traveled in locating the submerged platform resulted in a significant effect of days $(F=26.48$, df $=9,261, p<0.001$ and $F=31.76, \mathrm{df}=9,252, p<0.001$ for both the latency and distance data, respectively). Neither the main effects of age or treatment, nor any interaction was significant for either the 1 month or the 3 month intervention. Similarly, a single $30 \mathrm{sec}$ probe trial revealed no significant effect of age, treatment, or age by treatment interaction. Thus, 1 month of elevated serum corticosterone had no effect on learning or memory in either age group.

In the 3 month treatment group, analysis of the latency (Fig. $2 a$ ) and distance (Fig. $2 b$ ) data revealed effects of corticosterone treatment $(F=8.41, \mathrm{df}=1,30, p<0.01$ and $F=6.86$, df $=$ $1,30, p<0.01$ for the latency and distance data, respectively), days $(F=22.82$, df $=9,270, p<0.001$ and $F=23.88$, df $=$ $9,270, p<0.001)$, and an agc $\times$ trcatment $\times$ days interaction $(F=1.95, \mathrm{df}=9,270, p<0.04$ and $F=2.07, \mathrm{df}=9,270, p<$ $0.03)$. Post hoc analysis revealed significant differences between mid-aged, corticosterone- and cholesterol-treated rats on days 5 and 6 for the latency measure and on days $5-7$ for the distance measure (see Fig. $2 a, b$ ). Neither the main effect of age nor any of the simple interactions were significant. The probe trial, per- 

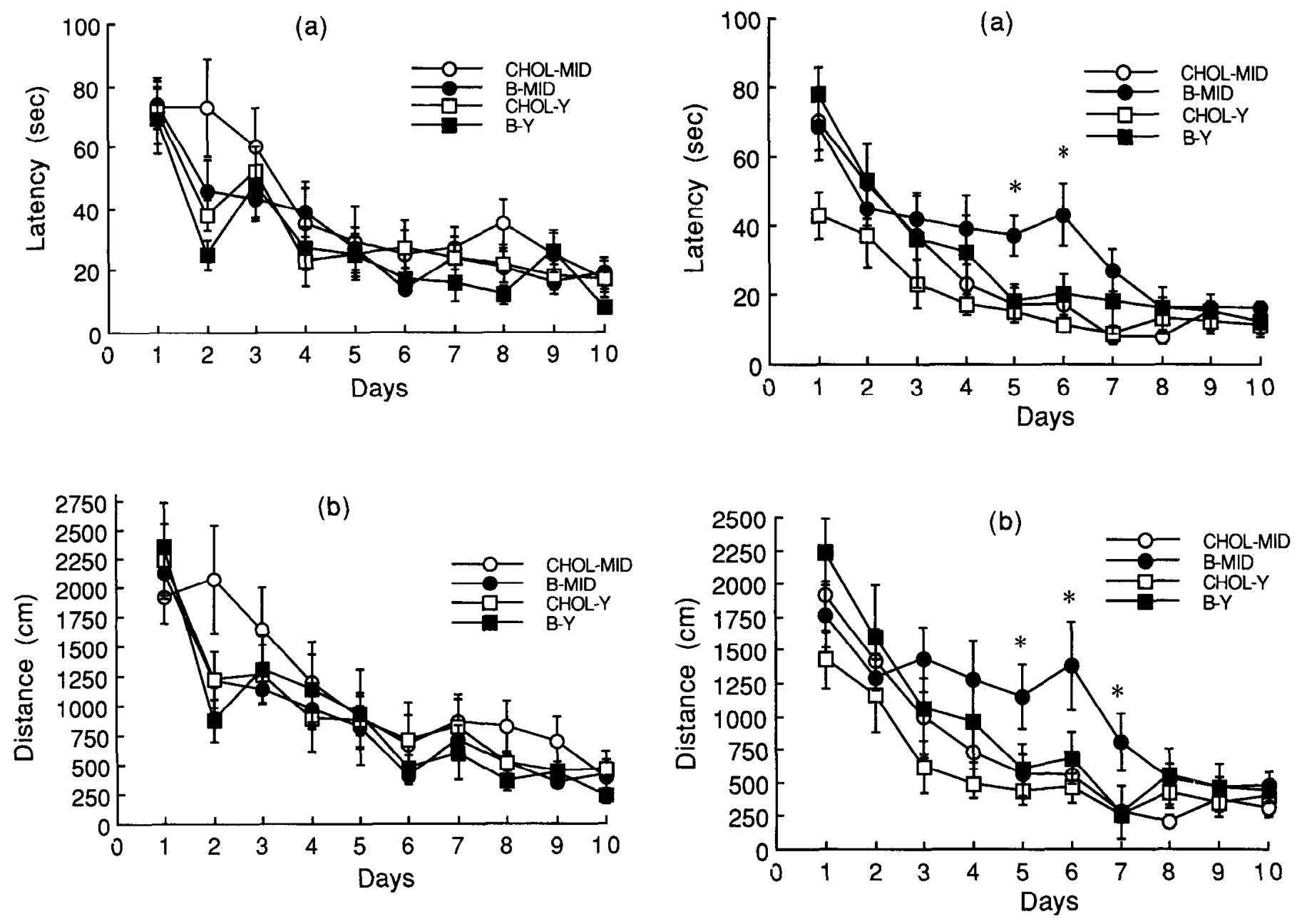

Figure 1. Mean ( \pm SEM) latency $(a)$ and distance $(b)$ to locate a submerged platform, following a 1 month treatment period in young adult $(Y)$ and mid-aged $(M I D)$, cholesterol $(C H O L)$, or corticosterone $(B)$ treated animals over days ( $n=8-10$ /per group).

formed at the end of testing, revealed no differences between the groups for either the 3 month treatment period. Thus, 3 month treatment with corticosterone retarded learning in midaged, but not young adult, animals.

Visible platform training produced swim latencies of under $10 \mathrm{sec}$ in all groups. There were no significant differences between any of the groups.

\section{Experiment 2: chronic stress and spatial learning in mid-aged rats}

Diurnal corticosterone levels. Diurnal corticosterone levels following 6 months of social stress or control conditions are shown in Figure 3. There was a significant group effect $(F=38.5$, df $=2,19, p<0.0001)$, as well as a significant effect of time of day $(F=13.0, \mathrm{df}=5,95, p<0.0001)$. Post hoc analysis of the simple main effects showed that the social stress group had significantly $(p<0.05)$ higher plasma corticosterone levels compared with the controls. Integrated corticosterone levels, calculated using the Trapezoidal Rule, showed that basal corticosterone levels were about twice as high in the stressed animals compared with controls (controls, $9.3 \pm 1.5 ; \mathrm{ADX}+\mathrm{B}, 6.8 \pm$ 1.2; social stress, $\left.16.0 \pm 1.8 \mu \mathrm{g} / \mathrm{dl} / \mathrm{hr}^{-1}\right)$. The largest effects of chronic social stress were observed in the samples taken during

Figure 2. Mean ( \pm SEM) latency $(a)$ and distance $(b)$ to locate a submerged platform, following a 3 month treatment period in young adult $(Y)$ and mid-aged $(M I D)$, cholesterol $(C H O L)$, or corticosterone $(B)$ treated animals over days $\left({ }^{*}, B-M I D\right.$ differs significantly from all other groups at $p<0.05 ; n=8--10 /$ per group).

the lights-on portion of the light cycle, when corticosteroid levels are usually lowest. This finding is consistent with several earlier reports (reviewed in Dallman et al., 1992) on the effects of more severe forms of chronic stress on basal corticosterone levels over the diurnal cycle, and serves to confirm the efficacy of this chronic stress manipulation.

Morris water maze. Figurc 4, $a$ and $b$, shows the mean latency and distance data (collapsed across blocks of two trials per day) for the Morris water maze. There was a significant effect of days $(F=46.9, \mathrm{df}=9,198, p<0.0001)$, a group effect $(F=3.2, \mathrm{df}$ $=2,22, p<0.01)$, and a group $\times$ days interaction $(F=3.2$, df $=18,198, p<0.0001$ ) on the latency measure. Post hoc analysis revealed that the social stress group was significantly impaired with respect to the control groups $(p<0.05)$ on days $2-6$ of testing (see Fig. $4 a$ ).

Analysis of the measures of distance traveled prior to locating the platform (see Fig. $4 b$ ) also showed a significant effect of days ( $F=35.3, \mathrm{df}=9,198, p<0.0001)$, a significant effect of group $(F=10.4, \mathrm{df}=18,198, p<0.001)$, and a significant group $\times$ days interaction $(F=2.2$, df $=18,198, p<0.01)$. Post hoc analysis showed that the social stress group differed significantly $(p<0.05)$ from controls on days $2-5$ of hidden platform learn- 


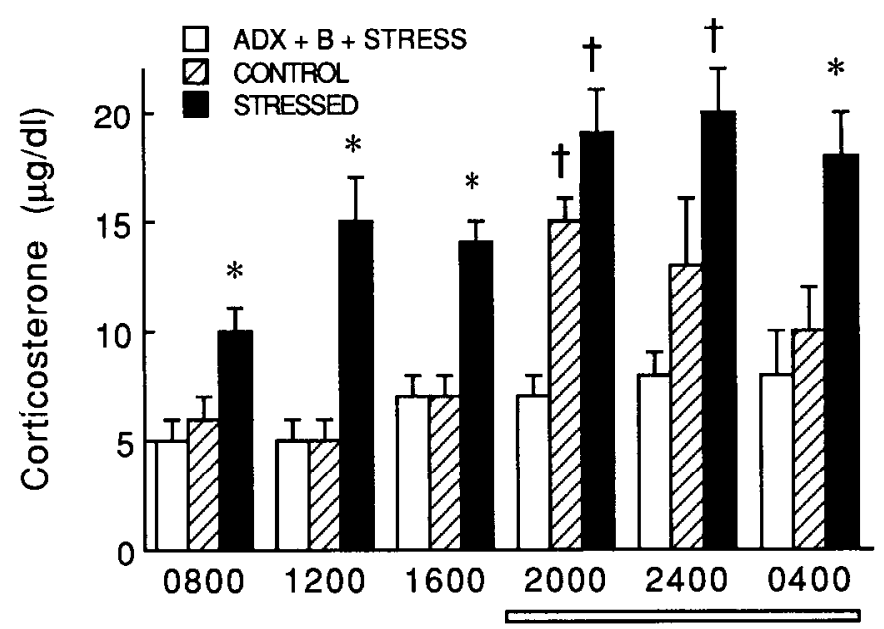

Time of Day

Figure 3. Mean ( \pm SEM) plasma corticosterone levels $(\mu \mathrm{g} / \mathrm{dl})$, taken at six consecutive $4 \mathrm{hr}$ intervals over the diurnal cycle, following 6 months of social stress (STRESSED), social stress + adrenalectomy with corticosterone replacement $(A D X+B+S T R E S S)$, or control conditions (CONTROL). *, corticosterone value that is significantly higher than other groups $(p<0.05) ; \dagger$, corticosterone value that is significantly higher than ADX + B + STRESS group; $n=8-9 /$ per group). The horizontal open bar indicates the period of lights out.

ing. No differences between groups were observed in subsequent visible platform training.

Experiment 3: chronic corticosterone, spatial learning, hippocampal plasticity, and morphology

Morris water maze. In experiment 3 , we examined young adult as well as mid-aged control animals. The performance of these animals did not differ on any measure; hence, the data were pooled into a single control group. Analysis of the latency and distance data (Fig. 5a,b) indicated a significant effect of corticosterone treatment $(F=5.97, \mathrm{df}=2,38, p<0.006$ and $F=$ $4.37, \mathrm{df}=2,38, p<0.02$ ), but no treatment $\times$ days interaction. Analysis of the simple main effect of treatment indicated that the high-B rats were significantly $(p<0.05)$ impaired with respect to both medium-B and controls from days 4 to 10 . In this experiment, the performance of the high-B animals never equaled that of the controls.

The results of the probe trial revealed that high-B rats spent significantly less time in the original training quadrant compared with medium-B and control animals (mean percentage of time in original quadrant: $25 \pm 4 \%, 40 \pm 4 \%$, and $41 \pm 4 \%$, respectively; $F=4.94$, df $=2,38, p<0.05$ ). In contrast, performance on the visually cued trials did not differ between the groups, with all rats finding the elevated platform in less than $12 \mathrm{sec}$.

Electrophysiology. Recordings were obtained from 11 control (four untreated young adults and seven mid-aged, cholesteroltreated controls), seven medium-B, and nine high-B animals. No differences were observed between the young and mid-aged control rats for any electrophysiological measurement. Thus, these two groups were pooled for subsequent analyses. For all the rats, the response to commissural stimulation recorded in the CA1 pyramidal cell layer consisted of a typical positive field EPSP with superimposed population spike (PS). Neither the
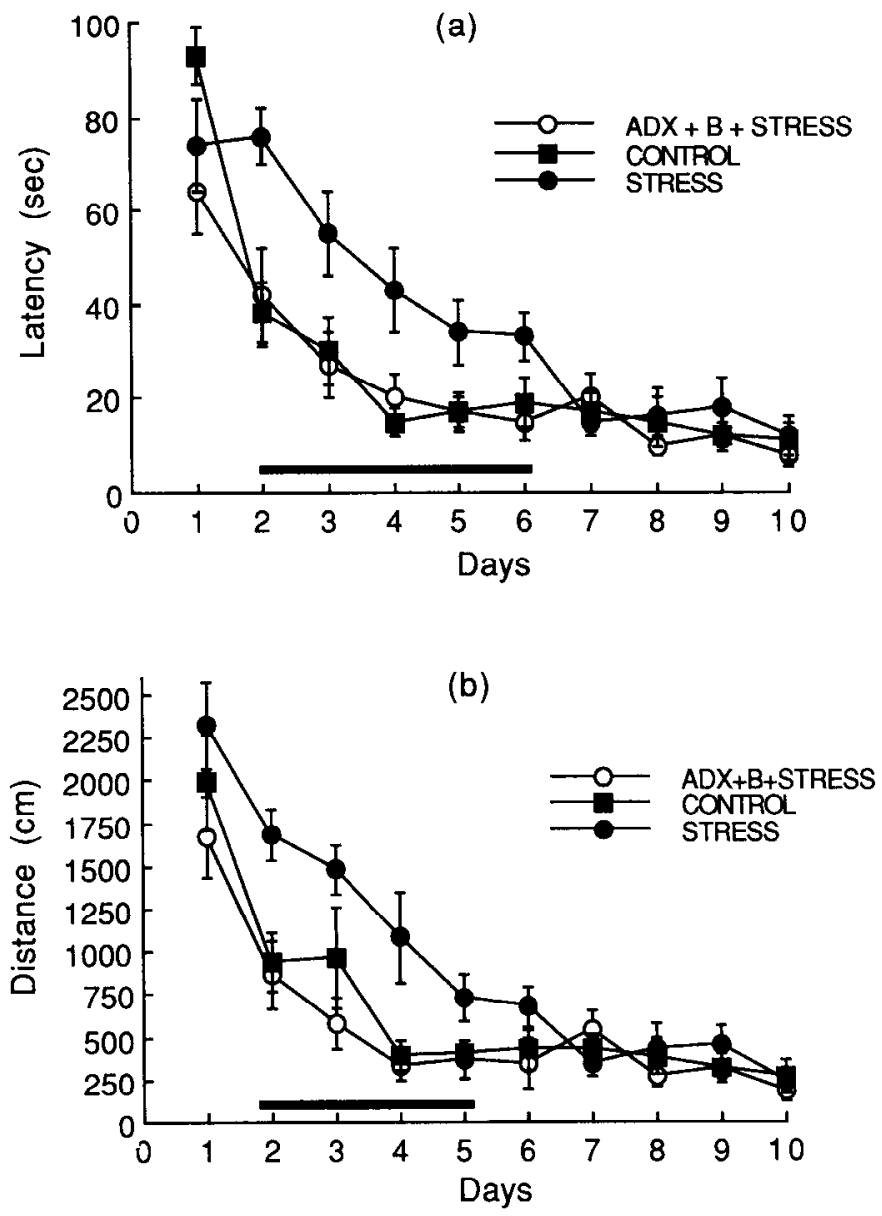

Figure 4. Mean ( \pm SEM) latency $(a)$ and distance $(b)$ to locate a submerged platform following 6 months of social stress (STRESS), social stress + adrenalectomy with corticosterone replacement $(A D X+B+$ STRESS), or control conditions (CONTROL). For each of the trials indicated by the horizontal bar, the performance of the STRESS animals differed significantly, $p<0.05$, from each of the other groups $(n=8-$ 9/per group).

baseline PS amplitude nor the stimulus intensity necessary to evoke the baseline PS differed among the groups $(p>0.2$ for both measures).

Following PB stimulation, the incidence of enhancement for individual rats was determined by comparing the response obtained in the last $5 \mathrm{~min}$ of the baseline period with those recorded during the interval 16-20 min after the PB train had been delivered (Student's $t$ test). Using this analysis, the incidence of enhancement following PB stimulation was, for control, 11 of 11 cases; medium-B, 5 of 7 cases; high-B, 6 of 9 cases. Figure 6 presents time course data for the three groups. Overall, there was a significant effect of treatment $(F=6.99$, df $=2,24, p<$ $0.004)$ and a treatment by time interaction $(F=4.75$, df 18,216 , $p<0.001$ ). Post hoc analyses showed that control animals exhibited significantly greater $\mathrm{PB}$ potentiation than both medium-B $(p<0.05)$ and high-B $(p<0.01)$ animals, which did not differ significantly from each other $(p>0.1)$. High-B animals also showed significantly less short-term plasticity (measured $1 \mathrm{~min}$ after $\mathrm{PB}$ simulation; $F=4.44, \mathrm{df}=2,24, p<0.02$ vs controls and medium-B). Hormone analysis on blood samples taken from the tail following the termination of the electrophysiological recording revealed that there were no differences between the groups in plasma corticosterone levels $(p>0.4)$. 

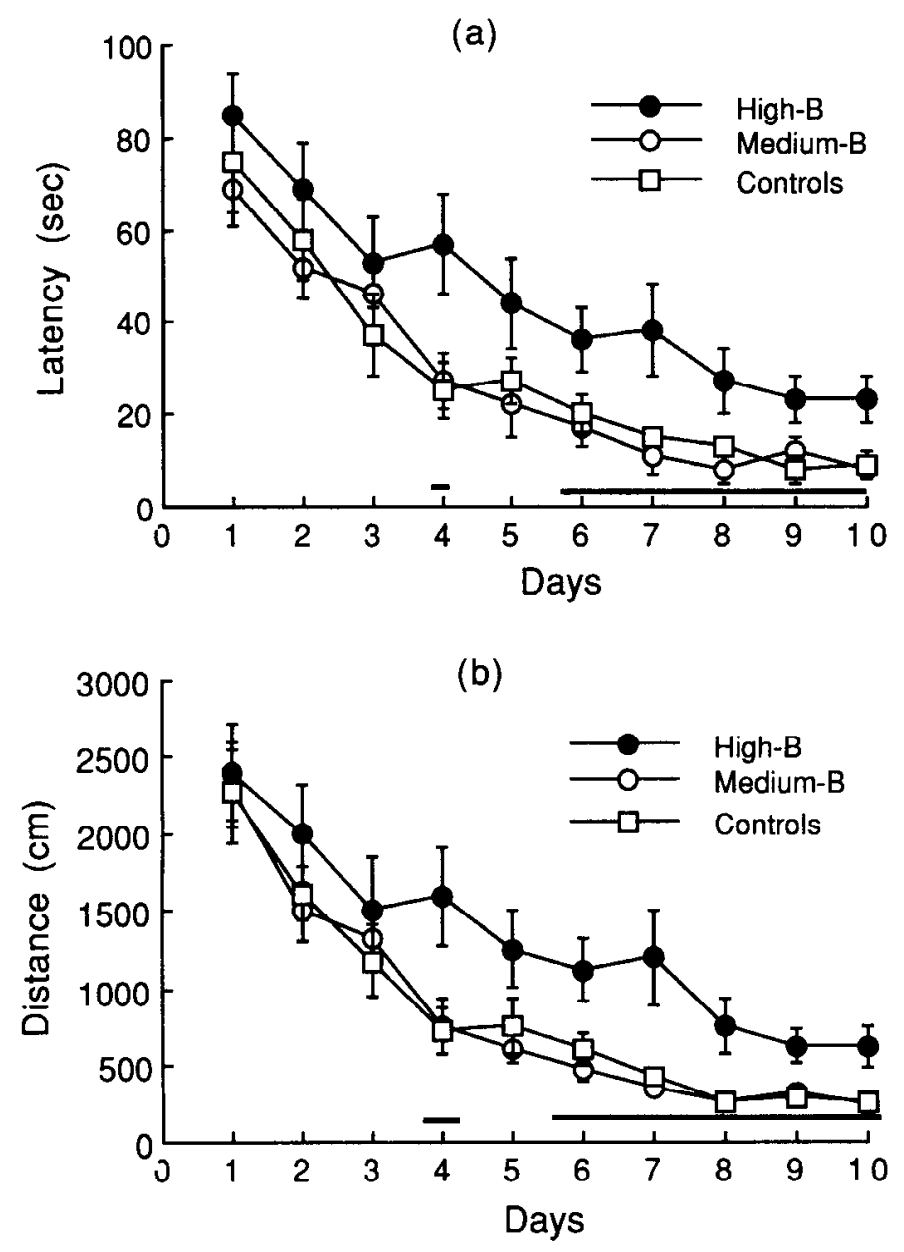

Figure 5. Mean ( \pm SEM) latency $(a)$ and distance $(b)$ to locate a submerged platform over testing days, in mid-aged animals treated for 3 months with a medium (Medium-B) or high (High-B) corticosterone levels, or controls. For each of the trials indicated by the horizontal bar, the performance of the high-B animals differed significantly, $p<0.05$, from each of the other groups ( $n=12-14$ /per group).

Hippocampal cell counting. Table 1 shows the mean number of corrected CA1 and CA3 neurons $/ 0.1 \mathrm{~mm}^{2}$ in mid-aged cholesterol-treated, medium-B and high-B rats randomly selected from experiment 3 . There were no significant differences between the treatment groups with respect to neuron density in either CA1 $(p>0.5)$ or CA3 $(p>0.15)$. Moreover, there were no group differences with respect to cell size $(p>0.8$ for CA1, $p>0.9$ for CA3). Finally, no differences in the overall dimensions of the cell fields were detected.

Table 1. Density and cell diameter of CA1 and CA3 hippocampal neurons in mid-aged cholesterol-treated, medium-B and high-B Rats

\begin{tabular}{lllll} 
& CA1 & \multicolumn{3}{c}{ CA3 } \\
\cline { 2 - 4 } & $\begin{array}{l}\text { Density } \\
\left(/ 0.1 \mathrm{~mm}^{2}\right)\end{array}$ & $\begin{array}{l}\text { Diameter } \\
(\mu \mathrm{m})\end{array}$ & $\begin{array}{l}\text { Density } \\
\left(/ 0.1 \mathrm{~mm}^{2}\right)\end{array}$ & $\begin{array}{l}\text { Diameter } \\
(\mu \mathrm{m})\end{array}$ \\
\hline Cholesterol & $40.0 \pm 0.9$ & $11.5 \pm 0.8$ & $22.5 \pm 0.8$ & $19.4 \pm 0.7$ \\
Medium-B & $38.0 \pm 2.4$ & $12.6 \pm 0.5$ & $24.5 \pm 1.1$ & $19.5 \pm 0.5$ \\
High-B & $40.1 \pm 0.9$ & $12.0 \pm 0.5$ & $22.3 \pm 0.6$ & $19.5 \pm 0.6$
\end{tabular}

Numbers are means ( \pm SEM) calculated from three to five sections per rat. $n=5$ rats for each group.

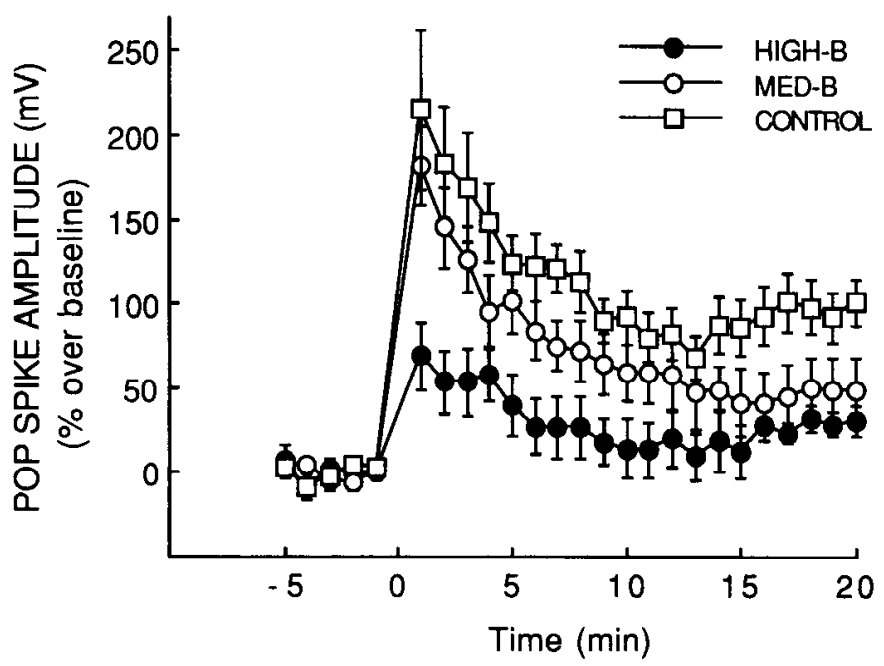

Figure 6. Mean $( \pm$ SEM) percentage change from baseline (time -5 to 0 ) of the population spike amplitude during the $5 \mathrm{~min}$ prior to and the 20 min following PB stimulation (time 0 ) in mid-aged animals treated for 3 months with a medium $(M E D-B)$ or high $(H I G H-B)$ corticosterone levels, or controls (CONTROL). Both medium-B and high-B rats showed significantly reduced $P B$ potentiation (measured $16-20$ minutes after the $\mathrm{PB}$ train) compared to the controls. High-B rats also had a significant reduction in short-term plasticity measured $1 \mathrm{~min}$ after PB stimulation. Each point indicates 1 min mean $( \pm$ SEM) values; $n=7-$ 11 per group.

\section{Discussion}

The primary findings of the present study were that 3 month, but not 1 month, treatment with corticostcronc, or a 6 month period of social stress, caused a lasting impairment in hippocampus-dependent spatial learning in mid-aged Long-Evans rats. In young adult rats, even the 3 month treatment regimen with high levels of corticosterone had no effect upon the acquisition of a spatial learning task compared with controls. Mid-aged rats with learning deficits also showed impaired hippocampal plasticity. Hippocampal pyramidal neuron density was unaffected by chronic corticosterone treatment, suggesting that both the learning and plasticity defects were present in the apparent absence of hippocampal neuron loss.

To date there have been somewhat contradictory findings in young, glucocorticoid-treated rats. Bardgett et al. (1994) reported that 2-month treatment with stress levels of corticosterone in Long-Evans rats impaired spontaneous alternation. However, in this study the treatment was continued throughout the behavioral testing period. Acutely elevated levels of corticosterone can interfere with learning (Bohus et al., 1970; De Weid and Croiset, 1991; Mondadori et al., 1992). Therefore, it is unclear whether the glucocorticoid mediated changes in behavior described by Bardgett et al. (1994) would be apparent in the absence of these elevated levels of corticosterone. In work more similar to our own, Dachir et al. (1993) observed delayed acquisition of a radial arm maze task by young F344 rats tested 1 week following the termination of a 9 week corticosterone treatment regimen. In this report, an effect of corticosterone treatment was no longer observed in the later stages of training. Similar findings were recently reported by Luine et al. (1994), who observed a transient impairment in radial arm maze acquisition in young Sprague-Dawley rats tested after 3 weeks of restraint stress. Thus, consensus on the behavioral sequelae of chronic corticosterone treatment in rats has yet to be reached. 
In contrast to the absence of a behavioral impairment in young rats, in the mid-aged rats a 3 month treatment period with stress-like levels of corticosterone significantly impaired spatial memory performance in the Morris water maze. While both mid-aged, corticosterone-treated (experiment 1) and the high-B (experiment 3 ) animals did exhibit a significant learning curve, they differed from age-matched controls during the middle days of training. However, the magnitude of the acquisition deficit differed between these two groups. For example, the glucocorticoid-treated rats in experiment 1 showed a slower rate of acquisition but eventually reached asymptotic performance that did not differ from controls. In contrast, in experiment 3 the performance of the high-B rats during both acquisition and retention (i.e., probe trial) never reached that of the controls. Although the reason for the difference between the two experiments is not entirely clear, it may be related to differing effects of the two types of corticosterone pellets employed. Although the average levels were not different, in experiment 1 the treatment resulted in slowly (over days) oscillating levels of corticosterone, while in experiment 3 the levels remained stable over the entire treatment period.

This study has provided evidence for the importance of elevated glucocorticoid titers in stress-induced cognitive impairments. Six months of chronic social stress produced spatial memory impairments in mid-aged rats. This finding is consistent with the report of Kerr et al. (1991), who reported that 6 months of chronic stress reduced hippocampal LTP. Adrenalectomy (with low-level corticosterone replacement) of animals chronically exposed to social stress completely blocked the effects of the stressor on spatial learning (see Fig. 4). These results provide direct evidence for the importance of clevated glucocorticoid levels in mediating the effects of chronic stress on hippocampal aging. These results are consistent with the previous study of Landfield et al. (1981) who demonstrated that adrenalectomy at mid-life (with low level corticosterone replacement) could attenuate hippocampal damage with aging.

The corticosterone-induced cognitive deficits observed in these experiments did not appear to have resulted from the loss of hippocampal pyramidal neurons. However, other authors have reported more subtle morphological changes in the hippocampus following corticosterone treatment (Wooley et al., 1990; Watanabe et al., 1992). Nevertheless, the absence of gross neuropathology in the present study offered an opportunity to explore further a potential mechanism underlying the behavioral impairment. While the suppressive effects of acute elevations of corticosterone upon hippocampal synaptic plasticity have been well documented (Bennett et al., 1991; Diamond et al., 1992; Pavlides, 1993), the consequences of long-term glucocorticoid administration (following the termination of the treatment) upon this measure had not been previously examined. Using a primed burst potentiation paradigm, we found that prolonged exposure to elevated glucocorticoid levels dampened potentiation, and that the effect was generally dose related. Animals maintained on steroid levels that mimicked either elevated basal corticosterone (medium-B) or stress-like corticosterone levels (high-B) both showed impaired potentiation, and the effect was greatest in the high- $B$ animals. These data are consistent with previous in vivo and in vitro studies correlating PB potentiation or LTP with age-related memory deficits (Landfield and Lynch, 1977; Landfield et al., 1978a; Barnes, 1979; Moore et al., 1993).

Although a measurable behavioral deficit was not apparent in the medium-B animals, the glucocorticoids did have an effect on hippocampal function as measured electrophysiologically. This incongruity between the behavioral and electrophysiological findings might suggest that there is a threshold of plasticity necessary to observe intact performance in the Morris water maze and that this level of plasticity did not occur in the high-B rats. However, there was also a qualitative difference in the topography of the response between the two groups. While the early-phase, posttetanic potentiation (PTP) response of the medium-B rats was equivalent to controls, high-B rats demonstrated a nondecaying (i.e., flat) response to tetanic stimulation. The latter finding was similar to that described by Deupree et al. (1991), who reported a correspondence between impaired performance in the Morris water maze and significantly reduced PTP and LTP. Thus, based upon the "normal" PTP response observed in the medium-B rats, a behavioral deficit in the Morris water maze would not be expected, while the absence of an enhanced PTP response in the high-B rats would predict impaired performance. What is apparent from the $\mathrm{PB}$ potentiation study is that the Morris water maze may not be the most effective tool for assessing subtle changes arising from long-term glucocorticoid exposure, such as were seen at the level of hippocampal synaptic potentials. Our present results suggest that the degree of hippocampal impairment must be severe before a behavioral deficit becomes apparent.

What are the potential mechanisms that might explain the enduring effects of glucocorticoids upon synaptic plasticity? Since plasma corticosterone levels at the time of the electrophysiological recordings did not differ between the groups, the suppressive effects of acute elevation of glucocorticoids can be ruled out. Instcad, it is conccivable that prolonged cxposure to corticosterone initiates a chain of events that eventually result in the decreased excitability of hippocampal neurons. It has long been known that acute administration of glucocorticoids alters the excitability of hippocampal neurons (Pfaffet al., 1971; Michal, 1974; Reiheld et al., 1984; Vidal et al., 1986). More recently, Joëls and DeKloet $(1989,1990)$ have shown that glucocorticoid treatment increases the duration and amplitude of the calciummediated slow afterhyperpolarization (AHP) in hippocampal neurons. The AHP is a potent inhibitor of neuronal excitability (Alger and Nicoll, 1980; Schwartzkroin and Stafstrom, 1980). In addition, we have previously argued that the AHP may participate in regulating the induction of primed burst potentiation (Bennett et al., 1991). It is possible that prolonged administration of glucocorticoids could initiate a permanent alteration in the AHP.

An interesting feature of these studies was the presence of glucocorticoid-induced spatial memory deficits in the apparent absence of hippocampal neuron loss. Neither the number nor the size of the pyramidal neurons of $\mathrm{CA} 1$ or $\mathrm{CA} 3$ region, or the extent of the cell fields measured in the dorsal hippocampus, was affected by the corticosterone treatment. These findings are consistent with those described by Bardgett et al. (1994), who also used Long-Evans rats, but not Dachir et al. (1993), who used F344 rats. Thus, hippocampal neuron loss does not appear to be an inevitable consequence of chronically elevated glucocorticoid levels. However, the phenomenon of neuron loss may be strain specific. The absence of detectable changes in hippocampal morphology following chronic corticosterone treatment conflicts with the original findings of Sapolsky et al. (1985). However, these authors, like Dachir et al. (1993), employed F344 rats in their studies. Sapolsky et al. (1985) reported a 50\% 
mortality rate with glucocorticoid treatment. Using Long-Evans rats, we found no deaths due to the treatment. Thus, glucocorticoids appeared to be more toxic to the Fischer strain.

What factors might account for this apparent strain difference in susceptibility to glucocorticoid-induced neurotoxicity in F344 rats? A potential contributor could be related to calcium levels in these animals. Calcium homeostasis is of interest given the evidence demonstrating the cytotoxic effects of elevated intracellular calcium (Farber, 1981; Choi, 1988), and the demonstration that glucocorticoids can contribute to calcium dysregulation (Landfield et al., 1992). A study of McBroom and Weiss (1973) has shown that F344 rats exhibit a rapid and marked increase in cortical calcium levels late in life, suggesting that there is an age-related breakdown in brain calcium equilibrium in this strain. Although the issue has not been studied directly, one might hypothesize that this phenomenon is not as marked in Long-Evans rats. The link between elevaled glucocorticoids and increased extracellular calcium levels appears to be related to the activation of NMDA receptors by glutamate. Glucocorticoids inhibit glutamate uptake by glial cells (Virgin et al., 1991). Glial uptake represents a major route for elimination of glutamate from the synapse (Hertz et al., 1983). Therefore, elevated levels of glutamate in the presence of glucocorticoids could remove the voltage-gated $\mathrm{Mg}^{2+}$ block on the NMDA receptor, thereby resulting in a persistent influx of $\mathrm{Ca}^{2+}$ that could lead to neuron death. This hypothesis is supported by the observation that NMDA antagonists also block the endangerment of hippocampal neurons by glucocorticoids (Armanini et al., 1990).

These findings might also bear on another question that emerges from our data: Why are the behavioral and electrophysiological effects of chronic stress or elevated corticosterone levels more prominent in mid-aged rats than in young rats? Sapolsky et al. (1986) hypothesized that glucocorticoids promote ongoing neurotoxic processes, but alone are not toxic to hippocampal neurons. The question then concerns the nature of the ongoing neurotoxic process. There is emerging evidence that disruption of mitochondrial energy generation may play a central role in age-related cognitive dysfunction (Blass et al., 1988; Kish et al., 1992). We have recently shown that, in an animal model of this process, glucocorticoids significantly potentiate the effect of a mitochondrial metabolic inhibitor (Bennett et al., 1993). Thus, the interaction of stress hormones with an ongoing deterioration of mechanisms of energy metabolism could play a central role in the generation of age-related cognitive deficits.

\section{References}

Abercrombie M (1946) Estimation of nuclear population from microtome sections. Anat Rec 94:239-247.

Alger BE, Nicoll RA (1980) Epileptiform burst afterhyperpolarization: calcium-dependent potassium potential in hippocampal CA 1 pyramidal cells. Science 210:1122-1124.

Armanini M, Hutchins C, Stein BA, Sapolsky RM (1990) Glucocorticoid endangerment of hippocampal neurons is NMDA-receptor dependent. Brain Res 532:7-12.

Bardgett ME, Taylor GT, Csernansky JG, Newcomer JW, Nock B (1994) Chronic corticosterone treatment impairs spontaneous alternation in the rat. Behav Neural Biol 61:186-190.

Barnes CA (1979) Memory deficits associated with senescence: a neuropsychological and behavioral study in the rat. J Comp Physiol Psychol 93:74-104.

Baxter JD, Tyrell JB (1987) The adrenal cortex. In: Endocrinology and metabolism (Felig P, Baxter JD, Broadus AE, Frohman LA, eds), pp 385-511. New York: McGraw-Hill.
Bennett MC, Diamond DM, Fleshner M, Rose GM (1991) Serum corticosterone level predicts the magnitude of hippocampal primed burst potentiation and depression in urethane-anesthetized rats. Psychobiology 19:301-307.

Bennett MC, Lehman JC, Mlady GW, Rose GM (1993) Synergy between corticosterone and sodium azide in producing a place learning deficit in the Morris water maze. Soc Neurosci Abstr 19:188.

Bentson J, Reza M, Winter J, Wilson G (1978) Steroids and apparent cerebral atrophy on computed tomography scans. J Comput Assist Tomogr 2:16-23.

Benveniste H, Drejer J, Schousboe A, Diemer NH (1984) Elevation of the extracellular concentrations of glutamate and aspartate in rat hippocampus during transient cerebral ischemia monitored by intracerebral microdialysis. J Neurochem 43:1369-1374.

Blass JP, Sheu RK-F, Cedarbaum JM (1988) Energy metabolism in disorders of the nervous system. Rev Neurol 144:543-563.

Bohus B, Grubits J, Kovács G, Lissák K (1970) Effect of corticosteroids on passive avoidance behaviour of rats. Acta Physiol Acad Sci Hung 38:381-391.

Brett LP, Chong GS, Coyle S, Levine S (1983) The pituitary-adrenal response to novel stimulation and ether stress in young adult and aged rats. Neurobiol Aging 4:133-138.

Brindley DN, Rolland Y (1989) Possible connections between stress, diabetes, obesity, hypertension and altered lipoprotein metabolism that may result in atherosclerosis. Clin Sci 77:453-461.

Choi DW (1988) Calcium-mediated neurotoxicity: relationship to specific channel types and role in ischemic damage. Trends Neurosci 11: 465-46́9.

Coleman PD, Flood DG (1987) Neuron numbers and dendritic extent in normal aging and Alzheimer's disease. Neurobiol Aging 8:521545.

Dachir S, Kadar T, Robinzon B, Levy A (1993) Cognitive deficits induced in young rats by long-term corticosterone administration. Behav Neural Biol 60:103-109.

Dallman MF, Akana SF, Scribner KA, Bradbury MJ, Walker C-D, Strack AM, Cascio CS (1992) Stress, feedback and facilitation in the hypothalamo-pituitary-adrenal axis. J Neuroendocrinol 4:517526.

DeKosky ST, Scheff SW, Cotman CW (1984) Elevated corticosterone levels. A possible cause of reduced axon sprouting in aged animals. Neuroendocrinology 38:33-38.

Deupree DL, Turner DA, Watters CL (1991) Spatial performance correlates with in vitro potentiation in young and aged Fischer 344 rats. Brain Res 554:1-9.

De Wied D, Croiset G (1991) Stress modulation of learning and memory processes. Methods Achiev Exp Pathol 15:167-199.

Diamond DM, Bennett MC, Fleshner M, Rose GM (1992) Inverted-U relationship between the level of peripheral corticosterone and the magnitude of hippocampal primed burst potentiation. Hippocampus $2: 421-430$.

Farber JL (1981) The role of calcium in cell death. Life Sci 29:12891295.

Foy MR, Stanton ME, Levine S, Thompson RF (1987) Behavioral stress impairs long-term potentiation in rodent hippocampus. Behav Neural Biol 48:138-149.

Hall RC, Popkin MK, Stickney SK, Gardner ER (1979) Presentation of the steroid psychoses. J Nerv Ment Dis 167:229-236.

Hertz L, Kvamme E, McGeer E, Schousboe A (1983) Glutamine, glutamate, and GABA in the central nervous system. New York: Liss.

Hess GD, Riegle GD (1970) Adrenocortical responsiveness to stress and ACTH in aging rats. J Gerontol 25:354-358.

Issa AM, Rowe W, Gauthier S, Meaney MJ (1990) Hypothalamicpituitary-adrenal activity in aged, cognitively impaired and cognitively unimpaired rats. J Neurosci 10:3247-3254.

Joëls M, de Kloet ER (1989) Effects of glucocorticoids and norepinephrine on the excitability in the hippocampus. Science 245:15021505.

Joëls M, de Kloet ER (1990) Mineralocorticoid receptor-mediated changes in membrane properties of rat CAl pyramidal neurons in vitro. Proc Natl Acad Sci USA 87:4495-4498.

Kerr DS, Campbell LW, Applegate MD, Brodish A, Landfield PW (1991) Chronic stress-induced acceleration of electrophysiologic and morphometric biomarkers of hippocampal aging. J Neurosci 11:13161324.

Kish SJ, Bergeron C, Rajput A, Dozic S, Mastrogiacomo F, Chang L-J, 
Wilson JM, DiStefano LM, Norbrega JN (1992) Brain cytochrome oxidase in Alzheimer's disease. J Neurochem 59:776-779.

Krey LC, Lu KH, Bulter WR, Hotchkiss J, Piva F, Knobil E (1975) Surgical disconnection of the medial basal hypothalamus and pituitary function in the rhesus monkey. II. GH and cortisol secretion. Endocrinology 96:1088-1093.

Landfield PW, Lynch G (1977) Impaired monosynaptic potentiation in in vitro hippocampal slices from aged, memory-deficient rats. $J$ Gerontol 32:523-533.

Landfield PW, McGaugh JL, Lynch G (1978a) Impaired synaptic potentiation in the hippocampus of aged, memory-deficient rats. Brain Res 150:85-101.

Landfield PW, Waymire IC, Lynch G (1978b) Hippocampal aging and adrenocorticoids: quantitative correlations. Science 202:10981102.

Landfield PW, Baskin RK, Pitler TA (1981) Brain aging correlates: retardation by hormonal pharmacological treatments. Science 214: 581-584.

Landfield PW, Thibault O, Mazzanti ML, Porter NM, Kerr DS (1992) Mechanisms of neuronal death in brain aging and Alzheimer's disease: role of endocrine-mediated calcium dyshomeostasis. J Neurobiol 23: $1247-1260$.

Ling MH, Perry PJ, Tsuang MT (1981) Side effects of corticosteroid therapy. Psychiatric aspects. Arch Gen Psychiatry 38:471-477.

Luine V, Villegas M, Martinez C, McEwen BS (1994) Repeated stress causes reversible impairments of spatial memory performance. Brain Res 639:167-170.

Madison DV, Malenka RC, Nicoll RA (1991) Mechanisms underlying long-term potentiation of synaptic transmission. Annu Rev Neurosci 14:379-397.

Martignini E, Costa A, Sinforiani E, Liuzzi A, Chiodini P, Mauri M, Bono G, Nappi G (1992) The brain as a target for adrenocortical steroids: cognitive implications. Psychoneuroendocrinology 17:343354.

McBroom MJ, Weiss AK (1973) A longitudinal and comparative study of the soft tissue calcium levels throughout the life-span of highly inbred rats. J Gerontol 28:143-151

Meaney MJ, Aitken DH, Viau V, Sharma S, Sarrieau A (1989) Neonatal handling alters adrenocortical negative feedback sensitivity and hippocampal type II glucocorticoid receptor binding in the rat. Neuroendocrinology 50:597-604.

Meaney MJ, Aitken DH, Sharma S, Viau V (1992) Basal ACTH, corticosterone and corticosterone-binding globulin levels over the diurnal cycle, and age-related changes in hippocampal type I and type II corticosteroid receptor binding capacity in young and aged, handled and nonhandled rats. Neuroendocrinology 55:204-213.

Michal EK (1974) Dexamethasone inhibits multi-unit activity in the rat hippocampus. Brain Res 65:180-183.

Momose KJ, Kjellberg RN, Kliman B (1971) High incidence of cortical atrophy of the cerebral and cerebellar hemispheres in Cushing's disease. Radiology 99:341-348.

Mondadori C, Ducret T, Häsler A (1992) Elevated corticosteroid levels block the memory-improving effects of nootropics and cholinomimetics. Psychpharmacology 108:11-15.

Moore CI, Browning MD, Rose GM (1993) Hippocampal plasticity induced by primed burst, but not LTP, stimulation is impaired in area $\mathrm{CA} 1$ of aged Fischer 344 rats. Hippocampus 3:57-66.

Mormede P, Lemaire V, Castanon N, Dulluc J, Laval M, Le Moal M (1990) Multiple neuroendocrine responses to chronic social stress: interaction between individual characteristics and situational factors. Physiol Behav 47:1099-1115.

Munck A, Guyre PM, Holbrook NJ (1984) Physiological functions of glucocorticoids in stress and their relation to pharmacological actions. Endocr Rev 5:25-44.

Okuno T, Ito M, Konishi Y, Yoshioka M, Nakano Y (1980) Cerebral atropy following ACTH therapy. J Comput Assist Tomogr 4:20-23.
Pavlides C, Watanabe Y, McEwen BS (1993) Effects of glucocorticoids on hippocampal long-term potentiation. Hippocampus 3:183-192.

Paxinos G, Watson C (1982) The rat brain in stereotaxic coordinates, 2d ed. New York: Academic.

Pfaff DW, Silva MT, Weiss JM (1971) Telemetered recording of hormone effects on hippocampal neurons. Science 172:394-395.

Reiheld CT, Teyler TJ, Vardaris RM (1984) Effects of corticosterone on the electrophysiology of hippocampal CA1 pyramidal cells in vitro. Brain Res Bull 12:349-353.

Rose GM, Dunwiddie TV (1986) Induction of hippocampal long-term potentiation using physiologically patterned stimulation. Neurosci Lett 69:244-248.

Rubinow DR, Post RM, Savard R, Gold PW (1984) Cortisol hypersecretion and cognitive impairment in depression. Arch Gen Psychiatry 41:279-283.

Sapolsky RM (1990) Glucocorticoids, hippocampal damage and the glutamatergic synapse. Prog Brain Res 86:13-23.

Sapolsky RM, Krey LC, McEwen BS (1983) The adrenocortical stressresponse in the aged male rat: impairment of recovery from stress. Exp Gerontol 18:55-64.

Sapolsky RM, Krey LC, McEwen BS (1985) Prolonged glucocorticoid exposure reduces hippocampal neuron number: implications for aging. J Neurosci 5:1222-1227.

Sapolsky RM, Krey LC, McEwen BS (1986) The neuroendocrinology of stress and aging: the glucocorticoid cascade hypothesis. Endocr Rev 7:284-301.

Schwartzkroin PA, Stafstrom CE (1980) Effects of EGTA on the calcium-activated afterhyperpolarization in hippocampal CA3 pyramidal cells. Science 210:1125-1126.

Sencar-Cupovic I, Milkovic S (1976) The development of sex differences in the adrenal morphology and responsiveness in stress of rats from birth to the end of life. Mech Ageing Dev 5:1-9.

Sonntag WE, Goliszek AG, Brodish A, Eldridge JC (1987) Diminished diurnal secretion of adrenocorticotropin (ACTH), but not corticosterone, in old male rats: possible relation to increased adrenal sensitivity to ACTH in vivo. Endocrinology 120:2308-2315.

Starkman MN, Schteingart DE, Schork MA (1981) Depressed mood and other psychiatric manifestations of Cushing's syndrome: relationship to hormone levels. Psychosom Med 43:3-18.

Starkman MN, Gebarski SS, Berent S, Schteingart DE (1992) Hippocampal formation volume, memory dysfunction, and cortisol levels in patients with Cushing's syndrome. Biol Psychiatry 32:756-765.

Tang F, Philips JG (1978) Some age-related changes in pituitaryadrenal function in the male laboratory rat. J Gerontol 33:377-382.

Varney NR, Alexander B, MacIndoe JH (1984) Reversible steroid dementia in patients without steroid psychosis. Am J Psychiatry 141: 369-372.

Vidal C, Jordan W, Zieglgänsberger W (1986) Corticosterone reduces the excitability of hippocampal pyramidal cells in vitro. Brain Res 383:54-59.

Virgin CE Jr, Ha TP, Packan DR, Tombaugh GC, Yang SH, Horner HC, Sapolsky RM (1991) Glucocorticoids inhibit glucose transport and glutamate uptake in hippocampal astrocytes: implications for glucocorticoid neurotoxicity. J Neurochem 57:1422-1428.

Watanabe Y, Gould E, Cameron H, McEwen BS (1992) Phenytoin prevents stress-/and corticosteroid-induced atrophy of CA3 pyramidal neurons. Hippocampus 4:431-436.

Whelan TB, Schteingart DE, Starkman MN, Smith A (1980) Neuropsychological deficits in Cushing's syndrome. J Nerv Ment Dis 168 : 753-757.

Wooley CA, Gould E, McEwen BS (1990) Exposure to cxcess glucocorticoids alters dendritic morphology of adult hippocampal pyramidal neurons. Brain Res 531:225-231. 